\title{
Argumentation scheme and shared online diagramming in case- based collaborative learning
}

\author{
J. Dowell, University College London, j.dowell@cs.ucl.ac.uk \\ M. Tscholl, University of Cambridge, mts483@cam.ac.uk \\ T. Gladisch, Dresden University of Technology, thomas.gladisch@inf.tu-dresden.de \\ M. Asgari-Targhi, University of Manchester, Marzieh.Asgari-Targhi@manchester.ac.uk
}

\begin{abstract}
Argumentation schemes describe patterns of reasoning in discourse. We report an investigation into whether the argumentation scheme known as inference to the best explanation' (IBE) captures the argumentation found in collaborative case-based learning. We examine the dialogue of three students working in an online learning environment as they attempt to explain the verdict in a legal case of medical negligence; the IDE scheme is clearly visible in the dialogue. We also report the exploratory development of shared argument diagramming tools that allow learners to draw their explanations while they discuss them. The tools passively reinforce the IBE argumentation scheme. Evaluation of the tools provided the clearest evidence to date that learners are able to integrate their shared online argument diagramming with their computer-mediated dialogue.
\end{abstract}

\section{Argumentation schemes and case-based collaborative learning}

Case-based learning is arguably the archetypal praxis for computer supported collaborative learning. Cases are representations of complex situations that call for explanation, analysis or resolution. The set of facts describing a situation will often be loosely structured, incomplete, and of varying reliability; several alternative explanations or solutions are usually possible. The case material discussed in this paper has all of these characteristics. It is an account of a law suit against a physician accused of negligence; a group of students struggles to explain the reasons for the judge's verdict in the case as part of their learning about medical law in professional practice. This making sense collectively of the facts of the case is characteristic of case based collaborative learning, and is increasingly regarded as a programmatic description of CSCL (Stahl, Koschmann $\&$ Suthers, 2006)

Learning through collaborative inquiry and explanation is an essentially inter-subjective phenomenon arising in the interactions between learners (Suthers, 2005; Suthers, Medina, Vatrapu, Dwyer, 2007). We should then regard the group of learners as a single cognitive system jointly constructing an explanation that resides in the semantic core of their discourse. More accurately, they may develop several competing explanations or converge progressively on a single explanation. Through their discussion, learners fit the facts within a structure that provides the relationships between those facts and abstracts their central meaning, rendering directly the explanation for the case (Tscholl and Dowell, 2008a). Individual learners possess distinct domain knowledge that is modified differentially by the collaborative development of explanations (Tscholl and Dowell, 2008b).

Critical argumentation is an analytical prism through which to view this process of meaning making in case-based collaborative learning. Its focus is the set of propositions in a discourse as a representation of the reasoning of the participants. It typically applies to dialectical situations where alternative conclusions are possible, but it is not limited to adversarial dialogues or disagreements. Occasionally, research is reported that finds that some particular dialogue or other contained few arguments, as indexed by the challenge or rebuttal moves it contains. However this is to use a lay notion of argument, rather than the sense of arguments and argumentation that we are concerned with here. Both the content of reasoning dialogues, and the rhetorical forms of those dialogues are the concern in critical argumentation.

People are adept at discourse as a process and no less so in a collaborative learning situation. They challenge and concede appropriately taking account of the structure of the discourse, turn taking, and previous contributions (Kuhn, Shaw \& Felton, 1997; Resnick et al 1993). When their communication is computer mediated and textual, those skills are arguably even more vital to sustaining a dialogue. But in contrast with their discursive skills, the arguments learners construct, particularly when using evidence, can be relatively inadequate (Schwarz \& Glassner, 2003). Those arguments may rely on non-justified beliefs rather than articulated reasons or theory, they often consist of detached reasons and may give no recognition of alternatives or rebuttal of counter-arguments (Kuhn, 1991). People frequently accept the plausibility of explanations without paying sufficient attention to their consistency with the available evidence (Brem \& Rips, 2000; Weinberger et al, 2006). These observations about the weakness of reasoning with evidence apply to both everyday and expert domains, as studies of jurors' reasoning (Carlson \& Russo, 2001) have clearly shown.

Critical argumentation attempts to understand the form and success of argumentation in discourse. It defines a typology of discourse types by relating them to the purposes of the discourse, the knowledge that the participants possess and come to possess in the discourse, and the methods they use. At a lower grain of 
analysis, critical argumentation concerns the form of arguments within distinct episodes in a discourse. It makes the important assumption that discourse contains stereotypical forms of reasoning or argumentation schemes. Argumentation schemes describe how discourse advances from one set of propositions to another, analogous to the advance from premises to conclusions in formal logic. They characterize the kinds of arguments typical of everyday conversation, arguments that subsequently can be overturned but that nevertheless provide useful heuristics for advancing understanding, particularly when information may be uncertain, unreliable or incomplete.

Many common kinds of argumentation schemes have been described; some 25 schemes are discussed in (Walton, 1996). For example, one of the most frequently cited schemes describes how expert opinion is incorporated into an argument: if a known expert asserts that some statement in their field of expertise is correct, then that statement should be regarded as correct. Argumentation schemes are useful for recognizing kinds of arguments, for recognizing the parts of arguments that are missing - such as the premises that people leave implicit, and they offer a basis for evaluating arguments. They have also been used for structuring interactions in multi-agent systems in artificial intelligence (Reed \& Walton, 2005). Associated with each kind of argumentation scheme is a set of critical questions to test arguments corresponding with the scheme. For example, one of the critical questions attached to the expert opinion scheme concerns consistency: do other experts agree that the statement is correct?

We can therefore speculate that case based collaborative learning is also associated with a particular argumentation scheme, and a strong candidate for it is 'inference to the best explanation' (IBE). This scheme applies to situations where an explanation needs to be formed and where alternative explanations are possible. IBE embodies a kind of reasoning that is abductive (Walton, 2005), sometimes also called retroductive, concerned with finding the most probable explanation for some observed event or object, in terms of the preceding conditions that caused it. Josephson and Josephson (1996) argued that much reasoning in ordinary life, and in science, medicine and law is of this kind; they contemplate whether abduction and planning are the primary functions of cognition.

Abductive reasoning, in contrast with formal deductive reasoning, is intrinsically creative, transforming partial knowledge into more complete and general knowledge. That knowledge must be tentative, rather than certain, given that it involves reasoning from consequent to antecedent. Peirce described abductive reasoning as "the only kind of reasoning which supplies new ideas, the only kind which is, in this sense, synthetic" (Peirce, 1997). Abductive reasoning therefore holds considerable promise as an account of how learning arises from explanation and problem solving. Abductive reasoning gets its name from the way in which explanations are lead by the data. Typically, an observation is made that is unexpected or requires explaining for some other reason; we recognize a hypothesis that explains the observation better than any other so we tentatively adopt it as our explanation.

Walton emphasizes the discursive context of IBE where explanation is driven by the need to find answers to successive questions:

" The best explanation is one that increases the understanding of a questioner as that individual moves forward through a search process. Of course, what increases understanding depends on the nature of the investigation... An abductive argument that is put forward by a proponent and meets the requirements for the scheme is to be evaluated in a given case with respect to how a respondent's critical questions are answered in a dialogue" (Walton, 2005, p. 206).

IBE should then be regarded as a process as much as a conclusion and at a larger scale than that of individual inferences. As a process on a larger scale, the IBE argumentation scheme describes the dialogue's lifecycle, extending from the dialogue setting, and formation of explanation attempts, through to the evaluation of explanations, and dialogue closure (Walton, 2005). A set of critical questions is associated with the evaluation phase: How adequate is the explanation relative to the alternatives? Have all the alternatives been found? How adequate is the explanation in itself for accounting for the given facts? How reliable are those facts?

To examine our assumption that IBE is the default argumentation scheme for case based collaborative reasoning, we will examine the dialogue taken from such a learning situation created in our research labs.

\section{An observation of collaborative learning through explaining a case of medical negligence}

We developed a case-based learning activity in medical law for undergraduate students taking a taught module on professional issues for clinicians. The setting for this learning activity was a collaborative learning environment constructed for the purpose. The environment provided shared access to a library of case materials, a synchronous chat system, and a shared note taking area. The students had already attended a lecture on the law of medical negligence and the learning experience we designed for our study substituted for the planned class work that would have involved a similar face-to-face discussion of cases. The learning experience we designed 
was focused on a case in which a general practitioner had been accused of negligence that it was claimed resulted in a patient suffering a stroke. We adapted the case for our purposes (Figure 1) from (Goldberg, 2000) to target the key issues of negligence: (i) whether the doctor's actions breached their duty of care to the patient, and (ii) whether actual harm was caused by a breach of duty of care. The relationship between these issues is potentially complex, and different qualities and forms of causation are possible. It is possible, for example, for a person to breach their duty of care, and therefore to be negligent, but for them not to be found liable for some harm that occurs because there were more significant factors involved more directly in causing the harm, because the harm was likely to have occurred regardless of the doctor's actions, etc. Hence the job of a court of law is to decide whether a person has been the victim of a negligent action and deserves compensation; disciplining a professional who has been negligent is the job of employers and professional bodies.

A 22-year old asian woman presented herself 3 times within a year at her General Practitioner's practice, with the intention of starting contraception before her marriage. She was to be married on $30^{\text {th }}$ of November and was eager to start the contraception. She was warned that there were health risks associated with contraceptive pills. On the third visit, on $11^{\text {th }}$ of October, her blood pressure (BP) was taken and it was at 150/100 (higher than normal for a woman of her age). This high reading was taken by the GP (Dr. Shaw) as a symptom of 'white-coat hypertension' - anxiety caused by being in a doctor's presence which can, however, be indicative of a general tendency to hypertension. The next day she started the pill. She claims that within 3 weeks she returned to the GP complaining about headaches and feeling generally ill. She says the GP prescribed a medication for the headache. The GP claims this meeting did not take place and records of any meetings that occurred during this period were no longer available. A week later, the plaintiff was admitted to hospital suffering from numbness and difficulty in walking. Her blood pressure was read several times and was variously found to be at 170/110,110/60 and 140/110. She was diagnosed as having suffered a stroke.

Note: the statistical evidence does not link taking contraceptives with stroke, over the population as a whole.

Figure 1. Description of the Vadera vs Shaw case

A group of three undergraduate medical students participated in this case-based learning experience. The students were presented with the description of the 'Vadera vs Shaw' case and were asked to explain the judge's verdict of 'negligent but not liable'. The students approach this task by giving their opinion of the doctor's negligence; they don't refer directly to the judge at all. An extract from their dialogue is reproduced in Figure 2 and includes the timestamp of each contribution. The order of a small number of contributions (those where the $\{$ timestamp $\}$ is enclosed in curly brackets) has been changed to make clearer what appears to be the intended sequence of exchanges, disrupted by time-ordered, post-once chat system.

\section{Evidence for the IBE argumentation scheme in the dialogue}

The argumentation of this group has the characteristics of both inquiry and persuasion (Dowell and AsgariTarghi, 2008). But can the IBE argumentation scheme be recognized in the dialogue as a structure encompassing the contributions of the three learners and characterising the movement in the dialogue?

The students can be seen to move from one tentative view to another as they try to make sense of the case in relation to their understanding of the law of medical negligence. The first hypothesis they advance suggests that the general practitioner was careless but not negligent (14:23:45), considering the fact that the GP ignored the unusually high blood pressure reading. When additional facts are then considered this hypothesis becomes less sustainable, for example, the new fact introduced by the students themselves that the normal protocol would have been to take additional readings of blood pressure. There is now a tentative and somewhat tacit agreement that the GP was negligent (14:26:41). Up to this point the learners were focused on whether or not the doctor's actions were negligent, or whether they were only careless (a careless act will not necessarily be negligent). The dialogue then considers the additional hypothesis about "the not liable part" of the verdict $(14: 27: 35)$. The first fact selected in relation to this hypothesis concerns the disputed consultation at which the GP is alleged to have ignored contra-indications to the prescription. David claims that this fact alone should decide the GP's liability. Gemma finally suggests a new hypothesis - that the case turns on what would have happened if the GP had not made the original prescription. This is a clear rejection of David's hypothesis and decisively re-frames the issue of the causal relationship between the doctor's actions and the actual harm suffered by the patient, which precisely applies the concept of liability. The students then look for other facts to 
confirm this counter-factual hypothesis and in doing so incorrectly interpret the statistical evidence: within the sub-population of hypertensives there is likely to be a significant correlation between strokes and the contraceptive pill which would then sanction the opposite hypothesis - that the GP was liable.

14:23:40 Gemma: What do u think about it david?

14:23:45 David: maybe the GP was a bit naive with teh white coat business

14:23:56 David: i mean 150/100?

14:24:05 Gemma: so $u$ would think that she is liable?

14:24:28 David: i would tend to say no

14:24:39 David: but I am evidently wrong here

14:24:55 Gemma: i don't think I'm understanding $\mathrm{u}$ ?

14:25:14 Gemma: which part of the verdict would $\mathrm{u}$ tend to disagree with?

14:25:35 David: well, i think the GP was maybe a little naive to say that such a high BP was white coat hypertension

$\{14: 25: 55\}$ Gemma : Yep i think i could agree with $u$ there...

14:25:46 David: but $i$ think it was a fair diagnosis

14:26:11 Gemma: so $u$ think she was right in prescribing the pill anyway?

14:26:18 Hywel: shouldn't he have repeated the test again at another time

14:26:21 David: Potentially

14:26:28 Gemma: Yeh thats what $i$ though

14:26:37: David: maybe she would have been better off taking repeated BP's, maybe at home?

$\{14: 26: 51\}$ : David: somewhere where the element of the doctors presence was reduced

14:26:41 Hywel: so he was negligent- he behaved irresponsibly

14:26:59 David: Or did he?
\{14:26:40 Gemma: And we don't know what kind of history the GP took

14:27:08 David: True

14:27:12 Hywel: i agree

14:27:35 Hywel: what about the not libale part?

14:27:42 David: I think that the crux here is whether the second meeting took place

14:28:01 Gemma: Yeh

$\{14: 28: 11\}$ David: if it did, and the GP fobbed her off, then definate case for negligance, not having followed up the symptoms etc.

$\{14: 28: 36\}$ David: but, if not then the GP wasnt to know about any adverse affects experienced

14:28:08: Hywel: what responsibility does the doctor have for keeping the records safe?

14:28:30 Gemma: yeh what about at the pharmacy, wouldn't there be a record of the prescription there?

14:29:26 Gemma: really the question is, would she have suffered the stroke if she hadn't been on the pill?

$\{14: 29: 33\}$ David: Indeed

\{14:29:41\} Hywel: apparently notstatistically anyway

14:31:21 David: so are we suggesting that the GP was not guilty of breach of duty?

14:31:24 Hywel: so if he took the same action as any other doctor would have (which the facts show that he did) he is not liable

14:31:40 Gemma: i say that because of the lack of statistical evidence linking the pill with stroke that the verdict is justified

14:31:40 David: Agreed

14:31:48 Hywel: Agreed

Figure 2. CMC group dialogue extract

In the students' dialogue the four critical questions appear implicitly: alternative explanations for different conclusions about Dr Shaw's negligence and liability are advanced and compared; each explanation is assessed to at least some degree against the case facts. The reliability of the facts is considered certainly in terms of their completeness. The students definitively conclude that the GP was not liable, although they identify just one fact as relevant to this hypothesis and they only identify part of the explanation connecting the relevant facts to this hypothesis. 
The students then have grasped the abstract distinction between negligence and liability but their use of it within the Vadera case is weak. David asserts that the key issue in deciding the GP's liability was whether Dr Shaw had ignored contra-indications to the prescription at a follow-on consultation. David appears to make several false assumptions here: that the GP could not be negligent for the original prescription; that negligence refers to acts of omission and not of commission; and that liability can be decided by reference to the doctor's actions without reference to whether those actions caused harm. Gemma then moves the discussion on to establish the counter-factual argument that will decide the matter. David acknowledges immediately the correctness of Gemma's argument as the central issue in deciding the GP's liability and abandons his own argument. David is learning to operationalise his concept of liability through the interaction.

This acquisition of new knowledge about medical negligence appears to be shaped by the IBE argumentation scheme. A tentative hypothesis is abandoned as new facts are considered, and a new hypothesis is tentatively accepted. As one hypothesis succeeds another, the conceptual differences underlying those hypotheses are exposed to each learner, modifying the knowledge each possesses about this domain (Tscholl \& Dowell, 2008a). The naïve concept of 'being to blame' comes to be replaced by the distinct concepts of 'being negligent' and 'being liable'. However the students' ability to interpret the concepts for the particular facts of the Vadera case remains weak.

The IBE scheme characterizes the argumentation of the three students and is useful in interpreting the learning outcomes of the dialogue. Discussion of the Vadera case by a different group, and that groups' discussion of a different case of medical negligence, are examined in Tscholl \& Dowell (2008b); again the dialogues can be seen to exhibit the IBE argumentation scheme which may well be characteristic of case-based collaborative learning. We now report the exploratory development of a collaborative case-based learning environment that supported shared argument diagramming with prompting of the IBE argumentation scheme.

\section{Online collaborative argument diagramming}

Graphical knowledge mapping tools are a common feature of learning environments designed for knowledge building in general and inquiry and explanation construction in particular. The list of celebrated exemplar systems includes Belvedere, CSILE, and SenseMaker (Suthers, 2003). These systems exploit a limited variety of representations, such as block and arrow graphs and structured lists for recording and analysing observations, hypotheses, backgrounder sources and evidential relations. These representations are accepted as encouraging more thorough inquiry, extended reflection, and more lucid reasoning. The learning value of knowledge mapping tools has been repeatedly demonstrated (see Kirschner et al, 2003); learners using the CSILE system were reported to "greatly surpass students in ordinary classrooms on measures of depth of learning and reflection, awareness of what they have learned or need to learn, and understanding of learning itself" (Scardamalia and Bereiter, 1994).

Collaborative argument diagramming provides one of the most intriguing and challenging prospects for computer supported collaborative learning environments. Such systems are those that enable a group of learners, each with their own interface, to collaboratively draw their reasoning during their discussion using synchronous groupware capabilities. Belvedere is amongst the best known of argument diagramming systems and has been examined in an online configuration, taking the original single user application program with graphing tools and source document browser/reader and augmenting them with a simple chat facility for synchronous text-based communication (Suthers 2003). Evaluations of the system with pairs of learners were comparative with the original face-to-face variant and focused on the influence of the argument graphs on the effectiveness of the collaboration.

Suther's studies provided unequivocal evidence that the argument diagrams play a greater role in the online collaboration condition; the online learners engaged in significantly more drawing activity and significantly less verbal communication activity. The greater focus on the diagram produced an increased presence in the dialogue of concepts that the software enforced, specifically on the relationships between individual facts and the categorization of statements as evidence or hypotheses. As the graph became a greater focus of the interactions between learners, new ideas were introduced directly into the diagram without first being shared verbally (an effect that could alternatively be interpreted as a breaking down of the collaboration). Suthers describes the chat transcripts as containing many examples of poorly coordinated activity, and in particular, disconnects between the activity in the workspace and the verbal activity in the chat.

A number of other efforts to assess online collaborative argument diagramming have been reported. A comparison of the online Belvedere system and the generic group meeting/authoring environment NetMeeting (available with earlier versions of Microsoft Windows) reported that the Belvedere dialogues were more conceptually oriented, though the results were confounded by the learners' difficulty in understanding the learning content (Veerman et al. 1999). Other studies with shared argument diagramming tools and synchronous communication facilities have reported that learners experienced difficulty with using the argument diagramming tools in combination with maintaining a dialogue; a comparison group working with the chat alone produced more successful arguments and achieved better learning outcomes (Baker, 2003; Baker et al. 
2003). Other recent reports of online collaborative argument diagramming have also described a system where a synchronous chat facility was provided separately from the argument diagramming tool (Munneke et al. 2007). The students were asked to debate an issue of ethics in relation to genetic technology and a comparison was made of the use of the shared argument diagramming system with a group text editor for composing summaries of the debate. The data appear to show that the diagrams supported a more satisfactory analysis but the dialogue between the students showed no benefit of the diagram.

There is in most of this work a consistent absence of a strong beneficial effect of the argument diagramming, with the possible exception of some of the effects found by Suthers. The absence of a stronger effect is puzzling and seems likely to be confounded by interface design factors as much as by collaboration or learning process factors. A particular feature of all the systems is the independence of the drawing and 'talking' facilities in the interfaces, which is experienced as a lack of integration by the learners: simply, they struggle to maintain a dialogue with the chat tool and managing a collaborative drawing activity in parallel only serves to make this harder.

What is needed then is a better integration of argument diagramming and verbal discourse. It is at this point that we return to the question of argumentation schemes. If, as we proposed earlier, there is a characteristic argumentation scheme for collaborative case-based learning, then that scheme characterizes both the dialogues and the contents of the argument diagrams. If this scheme can be introduced into the user interface then it has the potential as a vehicle for integrating the chat and the drawing tool. The Araucaria argument diagramming tool (Reed and Rowe, 2004) is an exemplar of how argumentation schemes may be used to support users in building argument diagrams, although it is neither a collaborative online tool, nor is it designed or used for learning. It is possible then that use of specific argumentation schemes could be reinforced in an online collaborative argument diagramming system to encourage a better integration of discussion with argument diagramming. We now describe our exploratory build of such a system in which the IBE argumentation scheme is reinforced.

\section{Reinforcement of the IBE argumentation scheme in collaborative argument diagramming online}

The COALA system (Cooperative Argumentation and Learning application) provides a shared diagramming tool, a chat facility for synchronous communication and a browsable library of case materials (Dowell and Gladisch, 2007)*. The argument diagramming tool uses a notation similar to Belvedere consisting of two node types. Data nodes contain facts and given information of the learning material or external sources, hypothesis nodes hold assumptions or conclusions that have been made by the users. The nodes can be linked to each other with different types of connections. These can either support or refute arguments. Furthermore, these connections can be annotated by the users to show specific criteria defining the connection. This typing of links is similar to the evaluation modifiers of Araucaria (Reed and Rowe, 2004), but allows for a more flexible arrangement of arguments in the diagramming area, since all nodes can be arranged freely and a single node can support and refute multiple nodes at once.

The diagram can only be modified by one user at a time and the annotator role can be requested and passed by and to any user. The annotator chooses how often to re-fresh their drawing to the rest of the group using a synchronise function, hence their drawing activity is not visible second by second by the other learners. COALA provides cut and paste from the case library and chat window into the contents of the diagram, to place quotations directly into the nodes of the diagram. Nodes can be flexibly re-positioned in the diagram and their links automatically re-drawn.

The screen shot of COALA in Figure 3 shows the case library, chat window containing the discussion between the users, and the argument diagram which is partially complete. The case material shown is again the Vadera vs Shaw case given to the group in the first study who used the simple online collaborative learning environment. The argument diagram consists of uniquely numbered data nodes (D) and hypothesis nodes $(\mathrm{H})$ linked by support relationships (single arrowhead) or refutation relationships (double-opposed arrowhead, the bold arrow indicating direction).

COALA attempts to integrate the diagramming and discussion activities through reinforcement of the IBE argumentation scheme. The reinforcement is provided by two features: the scheme checker, and the question asker, both contained in the scheme tool palette (shown in Figure 4). The palette is usually hidden from view and the user can make it visible using the controls in the window bar. The question asker reminds the learners of the critical questions that need to be asked of any argument corresponding with the IBE scheme. There are 4 such questions and the question asker feature allows learners to select one question and quote it directly in the chat system where it can be modified before sending. The scheme checker provides limited feedback on the conformance of the diagram syntactically with then IBE argumentation scheme, for example it advises users to consider additional hypotheses if appropriate. 

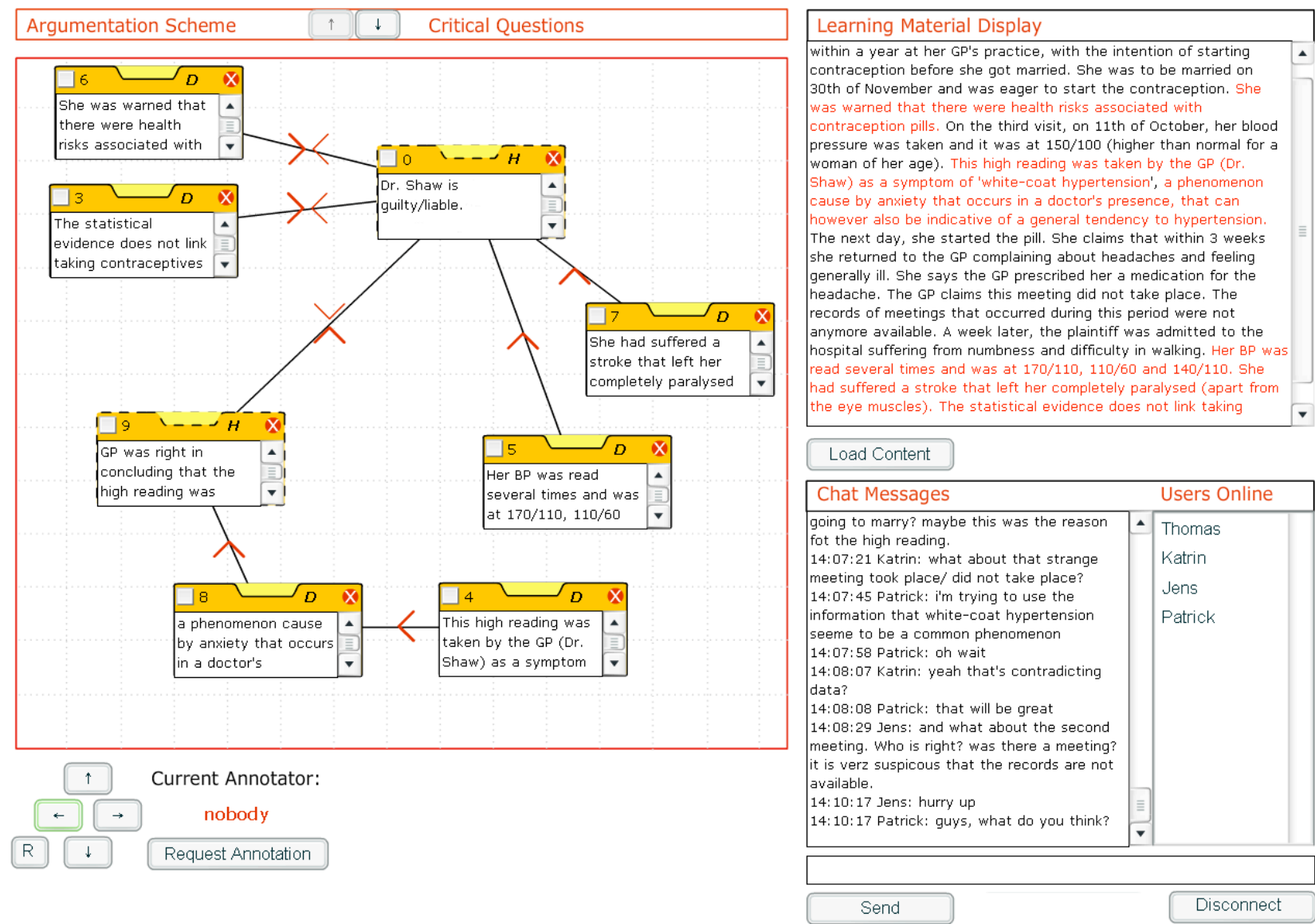

Whiteboard last updated 14:10:45 by Patrick

Figure 3. The COALA user interface

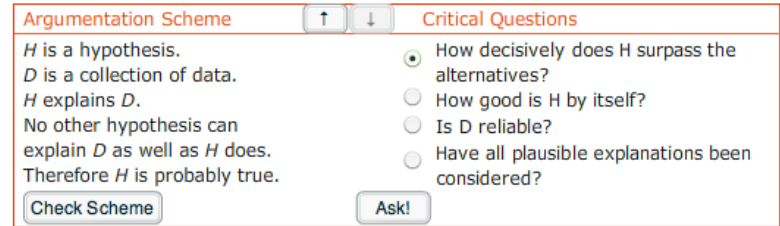

Figure 4. The argumentation scheme tool

In an evaluation of COALA with 4 groups of 3 users, a good level of consistency between the dialogues and diagrams was found (Dowell and Gladisch, 2007). The number of hypotheses introduced into the dialogues was consistent with the number of hypotheses appearing in the diagrams; similarly, the number of facts considered in the dialogues and the number appearing in the diagrams was also consistent. In post-session questionnaires, the learners indicated that the diagrams and dialogues were well integrated: the diagrams were broadly seen to be a fair or good representation of what had been discussed; the learners felt that with more practice with the tools they could produce even better diagrams. These results are in striking contrast to the reports of fracturing between dialogue and diagramming with previous online collaborative diagramming (Baker, Quignard et al. 2003; Suthers 2003 ).

However the results cannot be attributed to the reinforcement of the argumentation scheme tool. The learners in the evaluation (Dowell and Gladisch, 2007) made no explicit use of the tool for quoting the critical 
questions to the chat system, and the questions were not systematically visible in the dialogues either. In their questionnaire responses the users made clear that the critical questions were not useful. Three of the groups used the diagram checking tool to check their completed diagrams, although this did not prompt further modification of the diagrams.

The better integration of dialogue and diagram found in our study is most likely due to the direct manipulation features of the user interface, in particular, the facility for cutting and pasting text from the chat window and the case materials window into the diagram; this was well used by the learners and clearly helped them to integrate their discussion and diagramming. Simply making the system easier to use allowed the learners to devote more attention to the discussion and the task. Making the process of constructing the diagrams easier is unlikely to result in less thoughtful content in the diagrams, since the diagrams are created collectively. The integration of diagramming and discussion may have been affected by our choice of learning domain; medical negligence cases may be more amenable to diagramming than the learning cases given to learners in other studies that include pathogenetic explanations and ethical dilemmas.

The argumentation scheme tool was discretionary and limited, offering advice only to those users who sought it, and it may be that with re-design the tool would be better used. But we can also conjecture that a more active deployment of the argumentation scheme into the diagramming tool is needed to make the reinforcement felt. Suthers found that the presence of the argument diagramming encouraged his learners to refer to the concepts of the argument diagram representation, such as the relationships between the different pieces of evidence. A graphical representation of the argumentation scheme used as a framework or template in the argument diagramming tool would be likely to also encourage dialogue about the elements of the scheme, such as the adequacy of alternative hypotheses.

The development of COALA demonstrated convincingly that groups of online learners can diagram their arguments during collaborative case-based learning. The prior studies have elicited the argumentation scheme that characterizes such discussions. These findings are the correct basis on which to continue to explore ways of explicitly and actively reinforcing the argumentation scheme to facilitate argumentation in online collaborative learning environments.

*COALA is available freely via http://coala.gladisch.org/

\section{REFERENCES}

Baker, M. (2003) Computer-mediated argumentative interactions for the co-elaboration of scientific notions. In Andriessen J., Baker M. and Suthers D. (ed.s) Arguing to learn. Kluwer Academic: Netherlands.

Baker, M.J., Quignard, M., Lund, K. \& Séjourné, A. (2003). Computer-supported collaborative learning in the space of debate. In Proceedings of the International Conference on Computer Support for Collaborative Learning 2003. Dordrecht: Kluwer Academic Publishers.

Brem, S. K. \& Rips, L. J. (2000). Explanation and evidence in informal argument. Cognitive Science, 24(4), 573-604.

Carlson, K.A., \& Russo, J.E. (2001). Biased interpretation of evidence by mock jurors. Journal of Experimental Psychology: Applied, 7(2), 91-103.

Dowell, J. \& Asgari-Targhi, M. (2008) Learning by arguing about evidence and explanations. Argumentation, 22, 2, pp. 217-233.

Dowell, J. \& Gladisch, T. (2007) Design of argument diagramming tools for case-based group learning. In Wong W. (ed) Proceedings of the 14th European Conference on Cognitive Ergonomics. ACM Press.

Goldberg, R., 2000. The contraceptive pill, negligence and causation: views on Vadera v. Shaw, The Medical Law Review, volume 8, pages 316-338

Josephson, J. R. \& Josephson, S. G. (1996) Abductive Inference, Computation, Philosophy, Technology, Cambridge University Press.

Kirschner, P., Buckingham Shum, S. \& Carr C. (Eds.), (2003) Visualizing Argumentation: Software Tools for Collaborative and Educational Sense-Making. Springer-Verlag: London.

Kuhn, D. (1991). The skills of argument. Cambridge: Cambridge University Press.

Kuhn, D., Shaw, V., \& Felton, M. (1997). Effects of dyadic interaction on argumentive reasoning. Cognition and Instruction 15(3), 287-315.

Munneke, L., Andriessen, J., Kanselaar, G. \& Kirschner, P. (2007) Supporting interactive argumentation: Influence of representational tools on discussing a wicked problem Computers in Human Behaviour, 23, 3, 1072-1088

Peirce, C. S. (1997) Pragmatism as a principle and method of right thinking. In Patricia Ann Turrisi (Ed.), The 1903 Harvard Lectures on Pragmatism, State University of New York Press, Albany.

Reed, C. \& Walton D. (2005) Towards a formal and implemented model of argumentation schemes in agent communication, Autonomous Agents and Multi-Agent Systems, 11, 173-188 
Reed, C. \& Rowe G. (2004). Araucaria: Software for Argument Analysis, Diagramming and Representation. International Journal on Artificial Intelligence Tools 13( 4): 961 - 979.

Resnick, L. B., Salmon, M., Zeitz, C. M., Wathen, S. H. \& Holowchak, M. (1993). Reasoning in conversation. Cognition and Instruction, 11(3\&4), 347-364.

Scardamalia M. \& Bereiter C. (1994), Computer support for knowledge-building communities. The Journal of the Learning Sciences 3(3), 265-283.

Schwarz B. and Glassner A., (2003) The blind and the paralytic: supporting argumentation in everyday and scientific issues. In Andriessen J., Baker M and Suthers D. (ed.s) Arguing to learn. Kluwer Academic: Netherlands.

Stahl, G., Koschmann T. \& Suthers, D. (2006) CSCL: An historical perspective, in R. K. Sawyer (Ed.). (2006). Cambridge Handbook of the Learning Sciences. Cambridge, UK: Cambridge University Press

Suthers, D. (2003) Representational guidance for collaborative inquiry In Andriessen, J., Baker, M. and Suthers, D. (ed.s) Arguing to Learn Academic Publishers

Suthers, D. (2005). Technology affordances for intersubjective learning: A thematic agenda for CSCL. In Proceedings of the Computer Supported Collaborative Learning (CSCL) Conference 2005. New Bunswick: ISLS

Suthers, D., Medina, R., Vatrapu, R. \& Dwyer, N., (2007) Information sharing is incongruous with collaborative convergence: the case for interaction. In Proceedings of the Computer Supported Collaborative Learning (CSCL) Conference 2007. New Bunswick: ISLS

Tscholl M. \& Dowell J., (2008a) Characterising knowledge construction through a process analysis of dialogues. In Proceedings of the Eighth International Conference for the Learning Sciences - ICLS 2008. New Bunswick: ISLS

Tscholl M. \& Dowell J., (2008b) Analysing problem structuring in a collaborative explanation dialogue to capture conceptual change. In Proceedings of the 30th Annual Conference of the Cognitive Science Society. Austin, TX: Cognitive Science Society

Veerman, R., Andriessen, J. \& Kanselaar, G (1999). Collaborative learning through computer-mediated argumentation. In Proceedings of the 1999 conference on Computer support for collaborative learning.

Walton, D. (1996). Argumentation Schemes for Presumptive Reasoning, Mahwah, NJ: Lawrence Erlbaum Associates.

Walton D., (2005) Abductive Reasoning. Alabama University Press.

Weinberger, A., Clark, D., Erkens, G., Sampson, V., Stegmann, K., Janssen, J., Jaspers, J., Kanselaar, G. and Fischer, F. (2006) Argumentative Knowledge Construction in CSCL. In Proceedings of the International Conference of the Learning Sciences 2006. New Bunswick: ISLS

\section{Acknowledgments}

We acknowledge the support of the Economic and Social Research Council, award no. L328 253013 